\title{
Effect of Manganese and Potassium Humate on some Vegetative and Yield Parameters of Tomato Plant Lycopersicon esculentum Mill Grown in Plastic House
}

\author{
Hassan Hadi Hamza Al-Karwi ${ }^{\text {a }} \quad$ Hameed Kadhum Abd Al- Ameer $^{\mathrm{b}}$ \\ ${ }^{a, b}$ Al-Forat Al-Awsat Technical University, Technical College Mussayb \\ hassan2008hadi@googlemail.com hameed_almjadi@yahoo.com
}

Submission date:- 9/5/2018 Acceptance date:- 3/7/2018 Publication date:- 17/12/2018

Keywords: Manganese, Potassium humate, Tomato, Shahira.

\begin{abstract}
Afield experiment was conducted in a plastic house in Al-wattifiyah / Babylon Province during 2016/2017 season , soil texture was Loamy Sand with 3 levels of manganese element $\left(0,20\right.$, and $\left.40 \mathrm{mg} . \mathrm{L}^{-1}\right)$ using MnSO4.4H2O, and 4 levels of potassium humate $\left(0,10,20\right.$, and $\left.30 \mathrm{ml} . \mathrm{L}^{-1}\right)$, and their interaction on some vegetative and yield parameters of tomato plant Shahira variety. 4 spraying date among 20 days each, The experiment design was according to RCBD with 3 replicates, means were compared using L.S.D at 0.05 probability level.

The result show, the treatment (40 mg Mn. $\mathrm{L}^{-1}$ ) gave significant increases in plant height, total leaf No. , leaf area , plant dry matter, leaf content from chlorophyll, leaf dry matter percent, inflorescence number per plant, and No. of flowers per inflorescence, No. of fruit per plant, fruit weight, total yield of plant, and total yield per $\mathrm{m}^{2}$ gave high value was $212.65 \mathrm{~cm}, 42.7$ leaf , $172.6 \mathrm{ds}^{2}, 185.9 \mathrm{gm}, 45.03 \mathrm{spad}, 11.8$ inflorescence , 10.2 flowers , 47.6 fruits , $101.8 \mathrm{~g} \mathrm{~m}, 4.880 \mathrm{~kg}$, and $16.252 \mathrm{~kg} \cdot \mathrm{m}^{2}$ respectively. While potassium humate spraying treatment $\left(30 \mathrm{ml} . \mathrm{L}^{-1}\right)$ gave high value to same parameters above with $226.4 \mathrm{~cm}, 44.4$ leaf , $180.2 \mathrm{ds}^{2}, 196.4 \mathrm{gm}, 46.53 \mathrm{spad}, 11.8$ inflorescence, 10.3 flowers , 51 fruits , $105.5 \mathrm{gm}, 5.394 \mathrm{~kg}$, and $17.862 \mathrm{~kg} \cdot \mathrm{m}^{2}$ respectively . The interaction treatment (40 mgm Mn.L

${ }^{1}+30 \mathrm{ml} \cdot \mathrm{L}^{-1}$ potassium humate) gave the highest value of all parameters mentioned above.
\end{abstract}

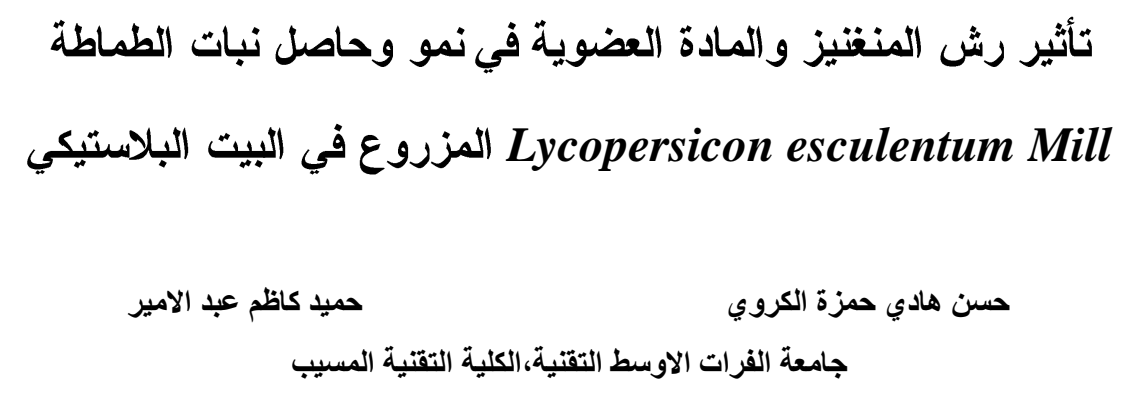

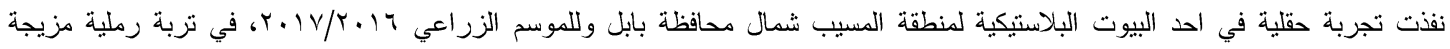

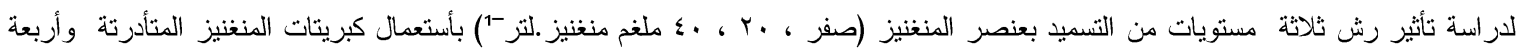

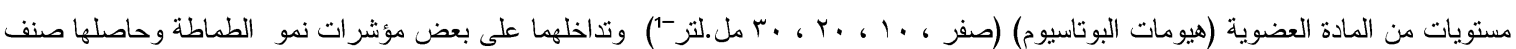


شهيرة وباربعة رشات كل ·r يوم، صمدت التجربة وفق تصميم القطاعات تامة التعشية RCBD وقورنت المتوسطات بأختبار اقل فرق معنوي \% \% و وبمستوى معنوية و

اثثارت النتائج الى تفوق معاملة رش المنغنيز بمستوى (•؛ ملغم.لتز -1) معنويا في زيادة متوسطات أطوال النبات ، اعداد الاوراق و المساحة

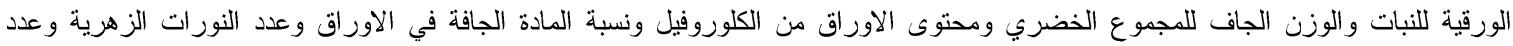

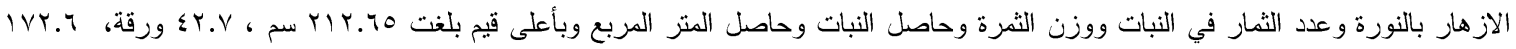

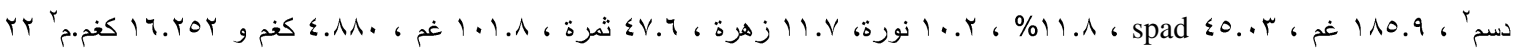

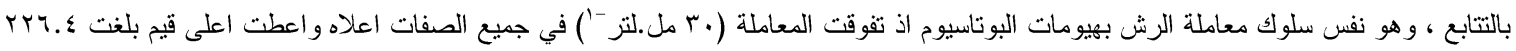

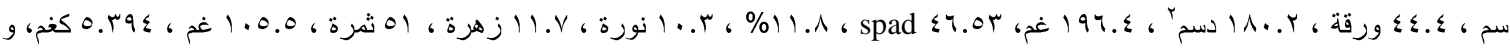

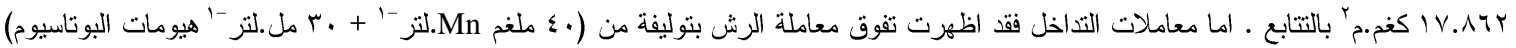
واعطائها اعلى القيم لجميع الصفات اعلاه.

الكلمات الدالة: منغنيز ، هيومات البوتاسيوم، الطماطة، شهيرة.

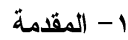

تعد الطماطة من محاصيل الخضر المهمة والرئيسة في العراق، وان ازدياد الطلب عليها دفع الكثير من المزارعين الى انتاجها تحت ظروف الزر اعة

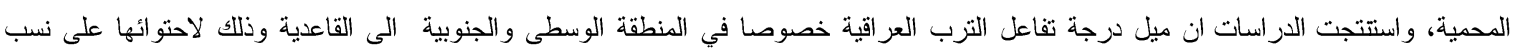

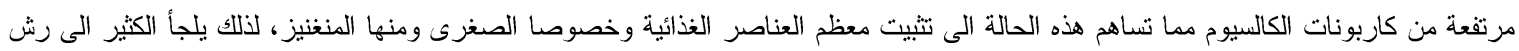

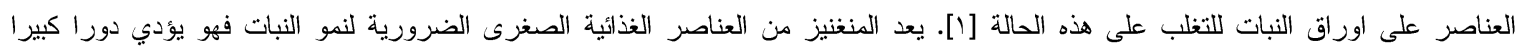

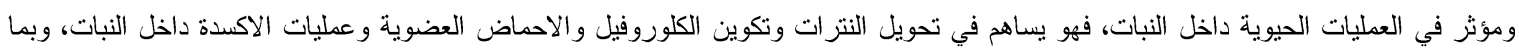

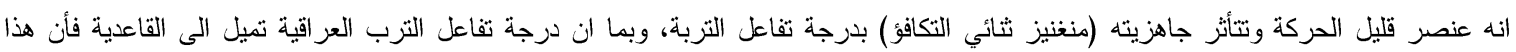

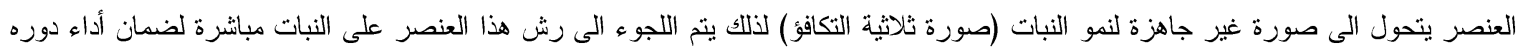

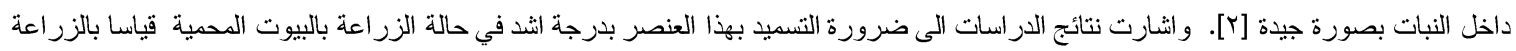

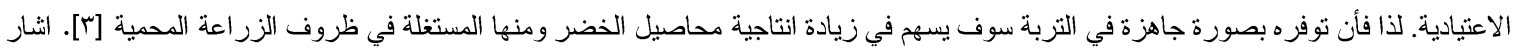

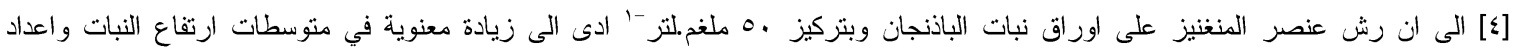

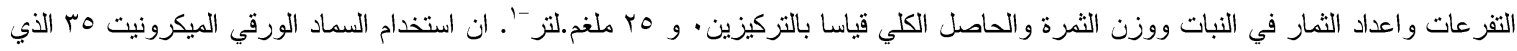

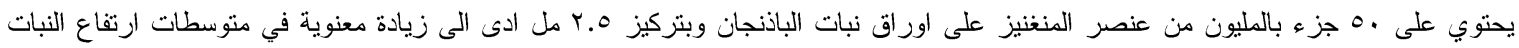

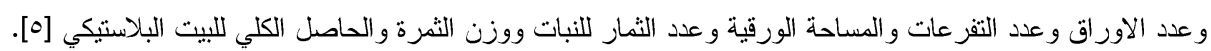

استخدمت المخصبات العضوية مثل (أحماض الهيومك) بتراكيز منخفضة لتحسين خواص التربة وتغذية النبات والإسراع في النمو وزيادة

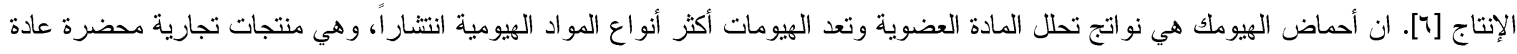
من الليونارديت Leonardite الذي يحوي • ب\% من الحوامض الهيومية والفولفية وعلى الارجح فان الهيومات التجارية تتكون من مزيج من الهيومات

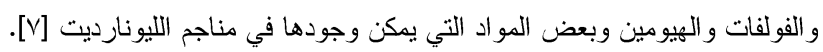
أن أحماض الهيومك لها تأثير ايجابي في امتصاص المغذيات من قبل النبات إذ تعمل على جاهزية العناصر و انتقالها خصوصاً المغذيات

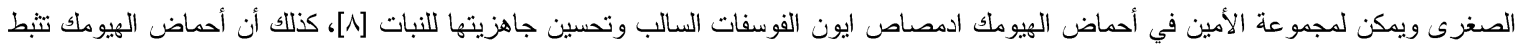

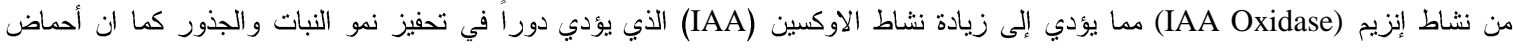
الهيومك تحسن من سعة مسك العناصر في التربة عن طريق ارتباطها بالصوديوم مما يساعد النبات على تحمل التزاكيز العالية لهذا العنصر والحماية

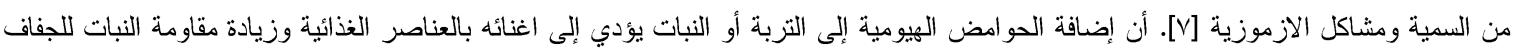

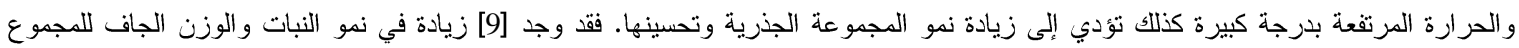

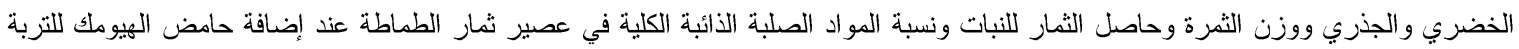

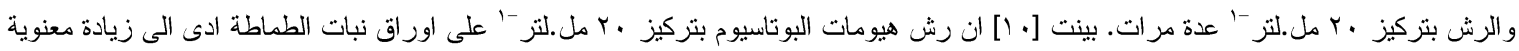
في متوسطات طول النبات وعدد الاوراق الكلي وعدد العناقيد الزهرية وعدد الازهار الكلي ونسبة العقد ووزن الثمرة وحاصل النبات ونسبة المواد الصلبة الذائبة الكلية في عصير الطماطة. 


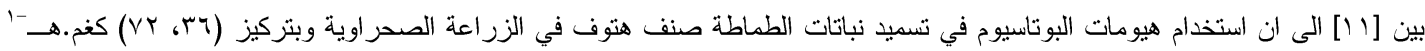

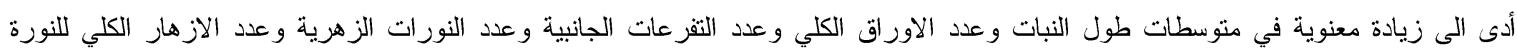
ووزن الثمرة وحاصل النبات والحاصل الكلي وفيتامين C وحموضة الثمار قياسا بمعاملة المقارنة عند عدم اضافة التانة السماد.

تهدف الدراسة الى معرفة تأثير عنصر المنغنيز والسماد العضوي السائل هيومات البوتاسيوم وتداخلها في نمو وحاصل نباتات الطماطة صنف شهيرة والمزروعة في البيت البلاستيكي غير المدفأة.

\section{r- ب المواد وطر ائق العمل}

نفذت التجربة في منطقة الوطيفية شمال محافظة بابل داخل البيت البلاستيكي لاحد مزارعي المنطقة بمساحة .0 بـ مب اثثاء الموسم الزراعي

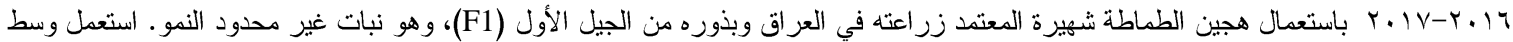

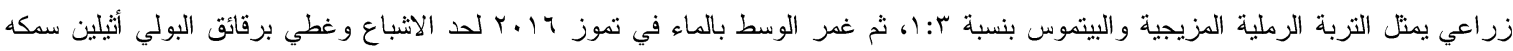

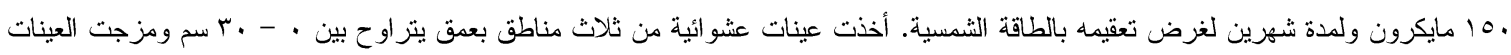

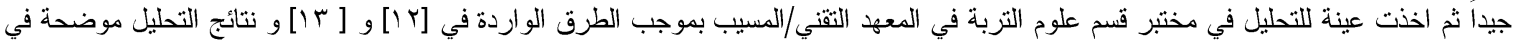
الجدول المان

جدول (1) مواصفات التربة المستعملة في التجربة

\begin{tabular}{|c|c|c|}
\hline القيمة & وحدة القياس & الصفة \\
\hline r.A & ديسي سيمنز •م -1 & التوصيل الكهربائي \\
\hline V.T & --- & تفاعل التربة \\
\hline $1 V .4$ & غم ـ كغم -1'تربة & المادة العضوية \\
\hline $10 . \varepsilon$ & سنتي مول ـ كغم'تربة & السعة التبادلية \\
\hline $1 V .0$ & غم ـ كغم -1 تزبة & كاربونات الكالسيوم \\
\hline 19.8 & \multirow{3}{*}{ سنتي مول. كغم'تربة } & النتزوجين الجاهز \\
\hline T. & & الفسفور الجاهز \\
\hline rی. 9 & & البوتاسيوم الجاهز \\
\hline $1 . r \Lambda$ & ميكاغر ام · م- مي & الكثافة الظاهرية \\
\hline T9Y.r & \multirow{3}{*}{ غم • كغم -1 تزبة } & 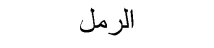 \\
\hline$r . r .0$ & & الغرين \\
\hline $1 \cdot \varepsilon . r$ & & الطين \\
\hline رملية مزيجة & & النسجة \\
\hline
\end{tabular}

قسمت أرض البيت البلاستيكي بعد التعقيم الى 0 مصاطب عرض كل منها ـ10 سم (مقسمة على عرض قناة المصطبة .0 سم، فيما كان

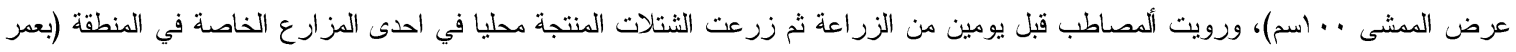

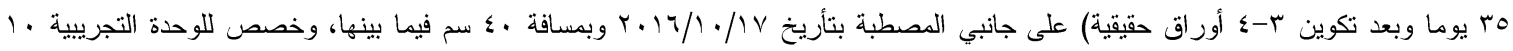

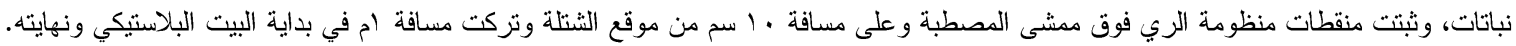

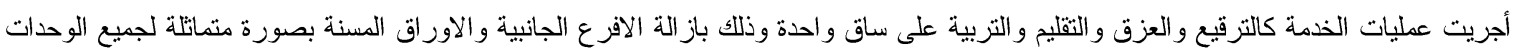

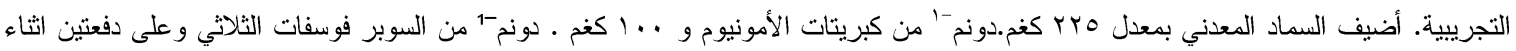

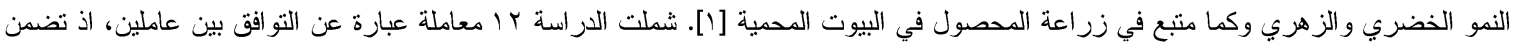




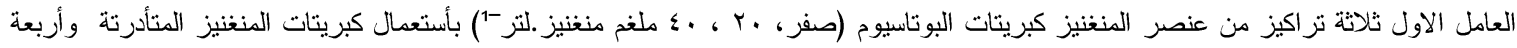

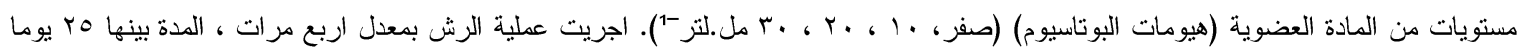

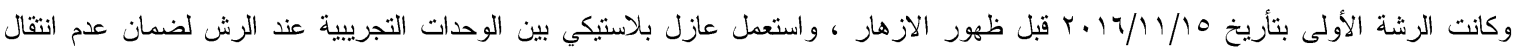

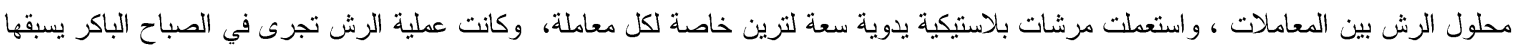

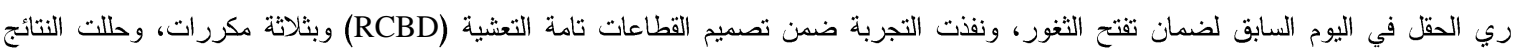

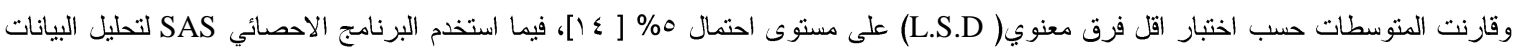

الصفات المدروسة/ مؤشرات النمو الخضري والزهري والحاصل :اختيرت ستة نباتات عشوائيا من كل وحدة تجريبية ووضعت علامات دالة عليها لغرض تسجبل البيانات لمؤشرات النمو الخضري الاتية: () طول النبات (سم): قيس هذا المؤشر في نهاية موسم النمو من منطقة اتصال الساق بالتزبة الى القمة النامية للنبات بوساطة الثريط المنزي. r) العدد الكلي للاور اق/نبات :حسب عدد الاوراق على الساق الرئيسة ولنهاية موسم النمو. ץ) المساحة الورقية للنبات( دسمץ): قيست المساحة الورقية للنباتات المعلمة لكل وحدة تجريبية وذلك بحساب مساحة ب أوراق مكتملة النمو مأخوذة من

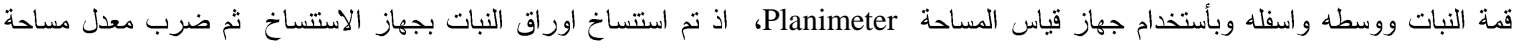
الورقة الو احدة في عدد اوراق النبات.

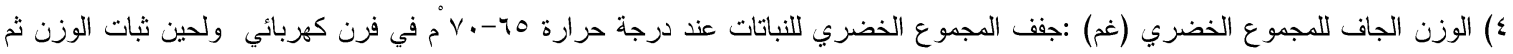
قيس الوزن الجاف بوساطة ميزان حساس. 0) محتوى الكلوروفيل (SPAD Unit): قدر بجهاز Chlorophyll meter نوع SPAD موقعيا وعلى النبات مباثرة وذلك بأخذ معدل ثلاث قراءات لكل ورقة.

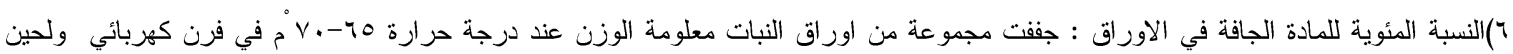
ثبات الوزن ثم قيس الوزن الجاف بوساطة ميزان حساس. V اعداد النور ات الزهرية للنبات : حسبت عددها معدل ستة نباتات لكل وحدة تجريبية ولنهاية موسم النمو. ^) اعداد الأزهار في النورة الواحدة : وحسب معدل ستة نباتات لكل وحدة تجريبية وعلى أساس عدد الازهار للنورات الزهرية الخمسة الاولى للنبات. 9) اعداد الثمار للنبات: وحسبت من حاصل جميع الجنيات (r ( جنية) وللنباتات المؤشرة وأخذ المتوسط لكل معاملة . • () وزن الثمرة غم : وزنت الثمار في كل جنية وأخذت المتوسط للجنيات ثم قسمت وزن الحاصل على عدد الثمار للحصول على متوسطوزن الثمرة للنبات. (1)(حاصل النبات كغم : جمعت اوزان الثمار للجنيات جميعها وللنباتات المؤشرة واستخرجت المتوسط لحاصل النبات الواحد . r ( ) حاصل المتر المربع كغم: وأستخرج حاصل المتر المربع الو احد على اساس حاصل النبات الواحد مضروبا في عدد النباتات في المتر المربع الو احد

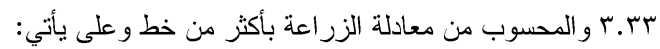
$\mathrm{x}=\mathrm{a}+\mathrm{b}(\mathrm{n}-1) / \mathrm{n} * \mathrm{c}$ حيث: x المساحة التي يشغلها النبات الواحد a المسافة بين كل مجمو عتين لخطي الزراعة (عرض ممشى المصطبة) المسافة بين خطي الزر اعة ضمن المجموعة الواحدة (عرض قناة المسطبة) b

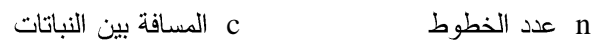


1. أبول النبات وعدد الاوراق للنبات

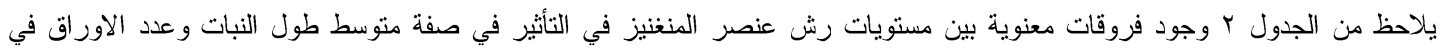

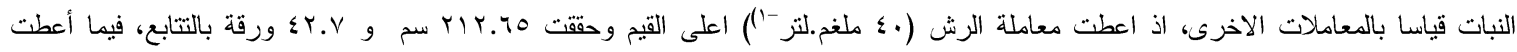

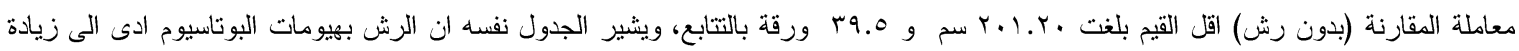

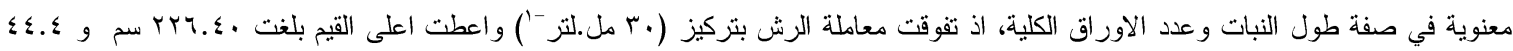

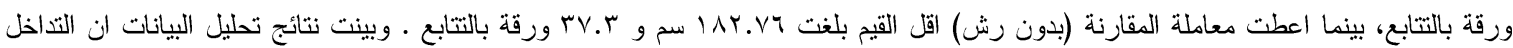

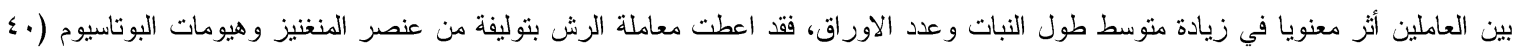

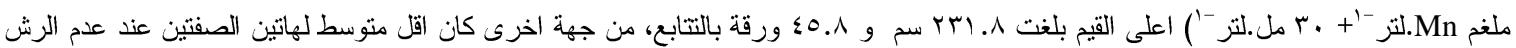

جدول (ץ) تأثير المنغنيز وهيومات البوتاسيوم وتاخلهما في متوسط طول النبات وعدد الاوراق الكلي

\begin{tabular}{|c|c|c|c|c|c|c|c|c|}
\hline \multicolumn{4}{|c|}{ العدد الكلي للاوراق.نبات-' } & \multicolumn{4}{|c|}{ طول النبات سم } & \multirow{3}{*}{ هلبولتاسيوم } \\
\hline \multicolumn{4}{|c|}{ تركيز المنغتيز ملغم .لتر-' } & \multicolumn{4}{|c|}{ تركيز المنغنيز ملغم.لتر-' } & \\
\hline المعدل & 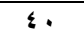 & $r$. & صفر & المعدل & $\varepsilon$. & r. & صفر & \\
\hline rV.r & $r 9.1$ & r..0 & ro.v & IAr.VT & 191.7 & $1 \wedge 1 . r$ & $1 \vee 0 . \varepsilon$ & صفر \\
\hline$\varepsilon \cdot .1$ & $\leqslant 1.9$ & $\varepsilon \cdot{ }^{\prime}$ & r^.1 & $r+1.7 r$ & $r \cdot V . r$ & Y. Y. & $190 . r$ & 1. \\
\hline$\leqslant Y_{0} \theta^{\circ}$ & $\varepsilon r . \varepsilon$ & $\varepsilon Y . r$ & $\leqslant 1 . \wedge$ & r17.9. & $r r_{\cdot} \cdot{ }^{*}$ & Y\^.1 & YIY. & $r$. \\
\hline \multirow[t]{2}{*}{$\varepsilon \varepsilon . \varepsilon$} & $\leqslant 0.1$ & $\varepsilon \varepsilon .1$ & $\varepsilon Y . \varepsilon$ & YYY.\&. & rr. & rYO.q & YYI.0 & $r$. \\
\hline & $\leqslant Y . V$ & $\varepsilon \cdot . \wedge$ & $r 9.0$ & & rir.to & $r \cdot 7.9 r$ & $r \cdot 1 . r \cdot$ & المعدل \\
\hline \multicolumn{2}{|c|}{ التداخل §؟Y.r } & $1 . \wedge \mathrm{V} \underline{\mathbf{H K}}$ & $1.62 \mathrm{Mn}$ & \multicolumn{2}{|c|}{ التداخل r.or } & $v .11 \underline{\mathrm{HK}}$ & $5.42 \mathrm{Mn}$ & LSD .05 \\
\hline
\end{tabular}

\section{r.بمعدل المساحة الورقية والمزن الجاف للمجموع الخضري للنبات}

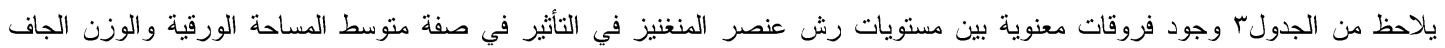

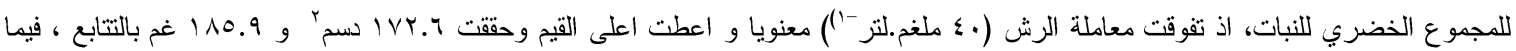

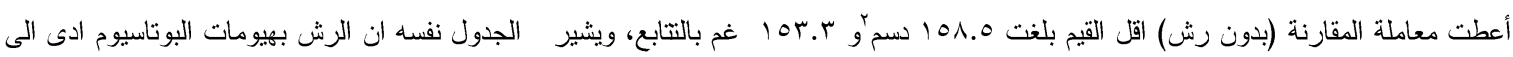

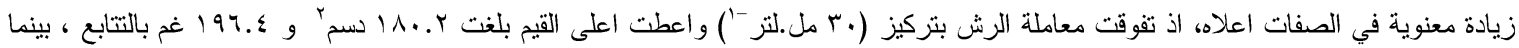

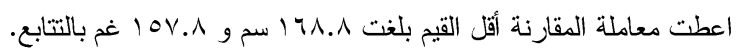


جدول (r) تأثير المنغنيز وهيومات البوتاسيوم وتداخلهما في متوسط المساحة الورقية و الوزن الجاف للنبات

\begin{tabular}{|c|c|c|c|c|c|c|c|c|}
\hline \multicolumn{4}{|c|}{ الوزن الجاف ـ نبات-1 غم } & \multicolumn{4}{|c|}{ المساحة الورقية دسمَ } & \multirow{3}{*}{ لمل هلبومات } \\
\hline \multicolumn{4}{|c|}{ تركيز المنغنيز ملفم .لتر -1 } & \multicolumn{4}{|c|}{ تركيز المنفنيز ملفم.لتر -1 } & \\
\hline المعدل & $\varepsilon$. & $r$. & صفر & المعدل & $\varepsilon$. & $r$. & صفر & \\
\hline $10 V .1$ & 178.8 & 104.4 & $1 \leqslant 9.1$ & lor.r & 174.8 & $1 \leqslant 9.1$ & $1 \leqslant V .7$ & صفر \\
\hline $178 . r$ & IVT.O & 174.9 & $17 \leq .7$ & $17 . .7$ & ITV.r & 109.1 & $100 . r$ & 1. \\
\hline $1 \Lambda \cdot r$ & 190.8 & IVr.A & $|v| . v$ & $174 .$. & $|V| . \varepsilon$ & $170 . r$ & 171.0 & $r$. \\
\hline \multirow[t]{2}{*}{197.8} & $r \cdot V . \varepsilon$ & 194.7 & 119.r & $1 \Lambda . . r$ & 119.8 & 111.7 & 179.8 & $\mu$. \\
\hline & 110.9 & 181.7 & 171.1 & & IVY.T & 174.9 & 101.0 & المعدل \\
\hline \multicolumn{2}{|c|}{ التاخل ^...r| } & ૫.VV $\underline{\mathbf{H}}$ & $5.89 \mathrm{Mn}$ & \multicolumn{2}{|c|}{ 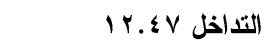 } & V.ro $\underline{\mathbf{H}}$ & $6.21 \mathrm{Mn}$ & LSD .05 \\
\hline
\end{tabular}

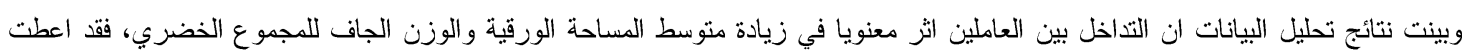

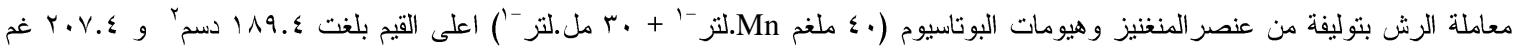

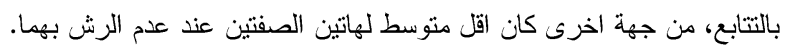

\section{r.r معدل محتوى الاوراق من الكلوروفيل ونسبة المادة الجافة في الاوراق}

يلاحظ من الجدول ؛ وجود فروقات معنوية بين مستويات رش عنصر المنغنيز في التأثير في صفة منوسط محتوى الاوراق من الكلوروفيل و ألوا

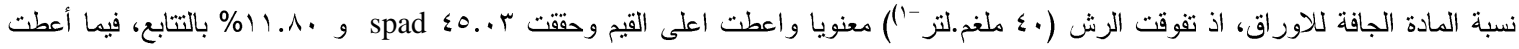

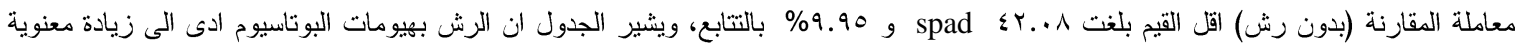

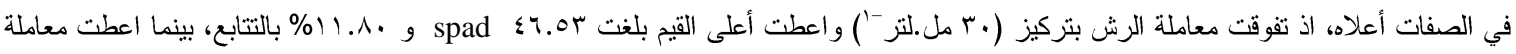

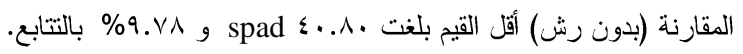

جدول (ء) تأثير المنفتيز وهيومات البوتاسيوم وتداخلهما في متوسط محتوى الكلوروفيل ونسبة المادة الجافة للاوراق

\begin{tabular}{|c|c|c|c|c|c|c|c|c|}
\hline \multicolumn{4}{|c|}{ المادة الجافة في الاوراق \% } & \multicolumn{4}{|c|}{ محتوى الكلوروفيل } & \multirow{3}{*}{ هلبومات } \\
\hline \multicolumn{4}{|c|}{ تركيز المنغنيز ملفم .لتر -1 } & \multicolumn{4}{|c|}{ تركيز المنفنيز ملفم.لتر -1 } & \\
\hline المعدل & ๑. & ro & صفر & 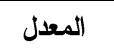 & $\varepsilon$. & $r$. & صفر & \\
\hline $9 . \vee \wedge$ & $1 . r r$ & q.v० & q.ro & $\varepsilon \cdot . \wedge$ & $£ 1.9$ & «1.r & rq.r & صفر \\
\hline 1.00 & $11.7 \mathrm{~V}$ & $1 \ldots 9$ & $9.1 \wedge$ & $\varepsilon Y . r V$ & $\varepsilon r .9$ & $\varepsilon \varphi . \vee$ & $\varepsilon . .0$ & 1. \\
\hline $11.1 \mathrm{r}$ & $1 r . .1$ & 11.99 & $1 . . r Y$ & $\varepsilon \varepsilon . \wedge V$ & ะ५.1 & $\{0.1$ & $\varepsilon r . \varepsilon$ & $r$. \\
\hline \multirow[t]{2}{*}{11.1.} & $1 T .14$ & $11 . \wedge \varepsilon$ & $1 . .2 \pi$ & $\varepsilon 7.0 r$ & $\{\wedge . r$ & 纟.. & $\{0.1$ & $r$. \\
\hline & 11.1. & $1 . .79$ & 9.90 & & $\varepsilon 0 . . r$ & $\varepsilon r . \wedge r$ & $\varepsilon r . . \wedge$ & المعدل \\
\hline \multicolumn{2}{|c|}{ التداخل 1.} & HK & $0.51 \underline{\mathrm{Mn}}$ & \multicolumn{2}{|c|}{ التداخل r.11 } & $1.29 \underline{\mathrm{H}}$ & $1.08 \mathrm{Mn}$ & LSD .05 \\
\hline
\end{tabular}


وبينت نتائج تحليل البيانات ان التداخل بين العاملين أنز معنويا في زيادة متوسط محتوى الكلوروفيل ونسبة المادة الجافة في الاوراق، فقد أعطت

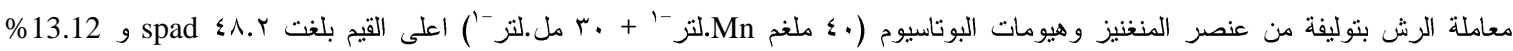

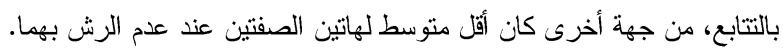

؛. كمعدل عدد النورات الزهرية وعدد الازهار بالنورة

يلاحظ من الجدول ه وجود فروقات معنوية بين مستويات رش عنصر المنغنيز في التأثير في صفة متوسط عدد النورات الزهرية وعدد الازهار

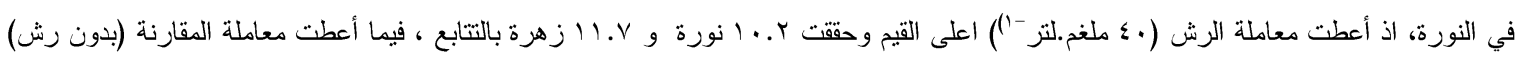

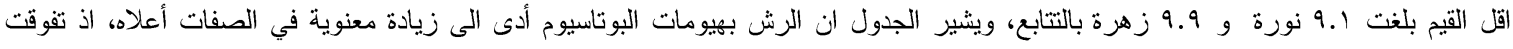

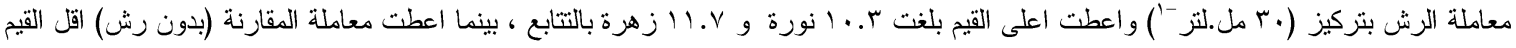

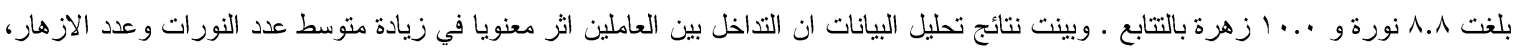

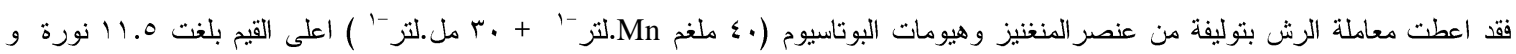

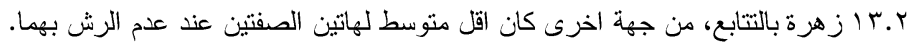

جدول (•) تأثير المنغنيز وهيومات البوتاسيوم وتداخلهما في متوسط عدد النورات وعدد الأزهار

\begin{tabular}{|c|c|c|c|c|c|c|c|c|}
\hline \multicolumn{4}{|c|}{ عدد الازهار.نورة-' } & \multicolumn{4}{|c|}{ عدد النورات ـ نبات-' } & \multirow{3}{*}{ لهوتاسيومات } \\
\hline \multicolumn{4}{|c|}{ تركيز المنغتيز ملفم للتر-' } & \multicolumn{4}{|c|}{ تركيز المنفيز ملفم.لتر-' } & \\
\hline المعدل & ๑. & ro & صفر & المعدل & $\varepsilon$. & r. & صفر & \\
\hline $1 \cdot . \cdot$ & $1 \cdot . v$ & 9.1 & 9.7 & $\Lambda . \Lambda$ & $9 . \%$ & $\Lambda . \vee$ & A. $\varepsilon$ & صفر \\
\hline $1 \cdot .4$ & 11.1 & $1 \cdot . r$ & $9 . \wedge$ & $9 . \xi$ & 9.7 & 9.0 & 9.1 & 1. \\
\hline $1 \cdot . v$ & 11.8 & $1 \cdot .0$ & 9.9 & 9.7 & $1 \cdot .1$ & 9.1 & $9 . r$ & $r$. \\
\hline \multirow[t]{3}{*}{$11 . v$} & IT.Y & 11.7 & $1 \cdot . r$ & $1 \cdot . r$ & 11.0 & 9.1 & 9.7 & $r$. \\
\hline & $11 . \mathrm{V}$ & $1 \cdot .0$ & 9.9 & & $1 \cdot . r$ & 9.0 & 9.1 & المعدل \\
\hline & 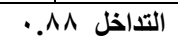 & $\underline{\text { HK }}$ & $0.41 \underline{\mathrm{Mn}}$ & \multicolumn{4}{|c|}{ 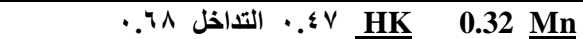 } & LSD .05 \\
\hline
\end{tabular}

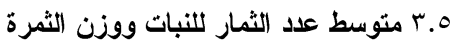

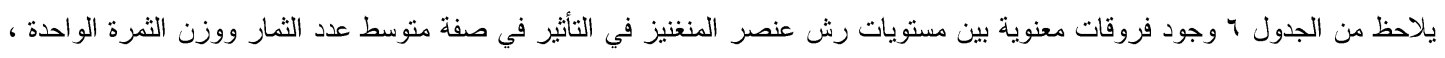

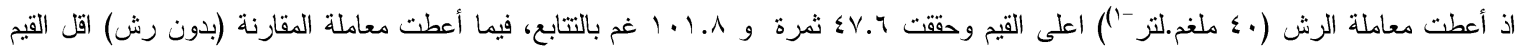

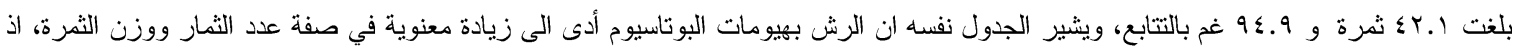

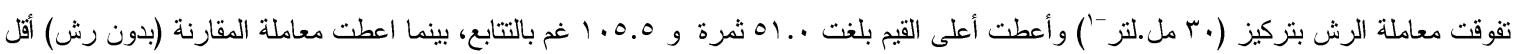

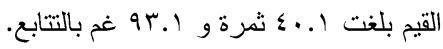


جدول (T) تأثير المنغتيز وهيومات البوتاسيوم وتداخلهما في متوسط عدد الثمار للنبات ووزن الثمرة

\begin{tabular}{|c|c|c|c|}
\hline \multicolumn{4}{|c|}{ وزن الثمرة غم } \\
\hline \multicolumn{4}{|c|}{ تركيز المنغنيز ملغم .لتر -1 } \\
\hline المعدل & o. & ro & صفر \\
\hline 94.1 & 90.1 & QY.A & $91 . \varepsilon$ \\
\hline $90 . r$ & Q४.० & $9 \leqslant .9$ & QT.r \\
\hline $99 .$. & $1 . T . \varepsilon$ & 91.7 & 90.1 \\
\hline \multirow[t]{2}{*}{1.0 .0} & 111.0 & $1.0 . r$ & 99.1 \\
\hline & 1.1 .1 & $9 \vee . \wedge$ & $9 \varepsilon .9$ \\
\hline \multicolumn{2}{|c|}{ التاخل ؟ ؟.9 } & HK & $.28 \mathrm{M}$ \\
\hline
\end{tabular}

\begin{tabular}{|c|c|c|c|c|}
\hline \multicolumn{4}{|c|}{ عدد الثمار .نبات-1' } & \multirow{3}{*}{ هل هلبومات } \\
\hline \multicolumn{4}{|c|}{ تركيز المنغنيز ملفم.لتر -1 } & \\
\hline 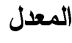 & $\varepsilon$. & r. & صفر & \\
\hline$\varepsilon \ldots 1$ & $\varepsilon \mu . \mu$ & rı.9 & rı.r & صفر \\
\hline «1.v & $\{\leqslant .1$ & $\{1.0$ & $r q . \varepsilon$ & 1. \\
\hline$\{0.0$ & $\{\wedge . \varepsilon$ & $\varepsilon \varepsilon . \wedge$ & $\varepsilon r . r$ & r. \\
\hline \multirow[t]{2}{*}{$01 .}$. & 0 ๑ . V & $0 . . v$ & $\varepsilon V .7$ & r. \\
\hline & $\{\vee .7$ & $\leq \pi .9$ & $\{r .1$ & المعدل \\
\hline \multicolumn{4}{|c|}{ 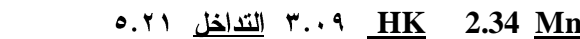 } & LSD .05 \\
\hline
\end{tabular}

وبينت نتائج تحليل الييانات ان التداخل بين العاملين أثز معنويا في زيادة منوسط عدد الثمار ووزن الثمرة، فقد أعطت معاملة الرش بتوليفة من

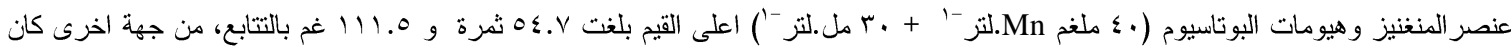
اقل متوسط لهاتين الصفتين عند عدم الرش بهما.

\section{Y Y.r متوسط حاصل النبات و الحاصل الكلي مP}

يلاحظ من الجدول V وجود فروقات معنوية بين مستويات رش عنصر المنغنيز في التأثير في صفة متوسط حاصل النبات الواحد وحاصل المتز

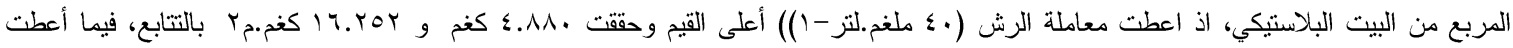

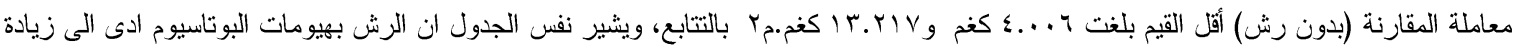

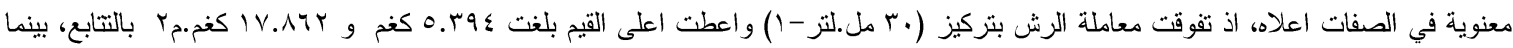

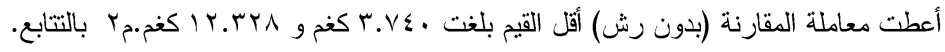

جدول (V) تأثثر المنفنيز وهيومات البوتاسيوم وتداخلهما في متوسط حاصل النبات وحاصل م־

\begin{tabular}{|c|c|c|c|c|c|c|c|c|}
\hline \multicolumn{4}{|c|}{ الحاصل الكلي كفم.م- } & \multicolumn{4}{|c|}{ حاصل النبات كغم } & \multirow{3}{*}{ 'لهيومات } \\
\hline \multicolumn{4}{|c|}{ تركيز المنغتيز ملفم .لتر -1 } & \multicolumn{4}{|c|}{ تركيز المنفنيز ملفم.لتر -1 } & \\
\hline المعدل & •. & ro & صفر & المعدل & $\varepsilon$. & r. & صفر & \\
\hline IY.TYA & Ir.vir & $11.91 \%$ & 11.04. & 3.740 & 4.118 & 3.610 & 3.491 & صفر \\
\hline $1 T .1 \leqslant \varepsilon$ & 18.419 & 17.990 & 19.111 & 3.970 & 4.300 & 3.938 & 3.672 & 1. \\
\hline $1 \varepsilon .9 r r$ & 17.778 & 1 1ะ.0V4 & 17.004 & 4.510 & 5.005 & 4.417 & 4.108 & r. \\
\hline \multirow[t]{2}{*}{ IV.ATY } & $r . . r_{1}$. & $1 V .7 \cdot Y$ & 10.780 & 5.394 & 6.099 & 5.334 & 4.750 & $\mu$. \\
\hline & 17.ror & $|\varepsilon . Y V|$ & IT.YIV & & 4.880 & 4.325 & 4.006 & المعدل \\
\hline \multicolumn{2}{|c|}{ التـاخل بـ ـ.ب } & Y HK & $1.89 \mathrm{Mn}$ & \multicolumn{2}{|c|}{ ألتداخل 99.. } &.. $\mathrm{V} \underline{\mathrm{H}}$ & $0.43 \underline{\mathrm{Mn}}$ & LSD .05 \\
\hline
\end{tabular}


ويبنت نتائج تحليل الييانات ان التداخل بين العاملين أثز معنويا في زيادة متوسط حاصل النبات وحاصل المتز المربع، فقد أعطت معاملة الرش بتوليفة

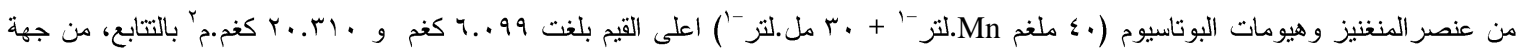

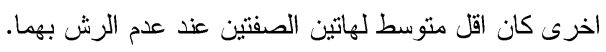

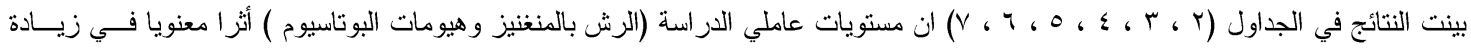

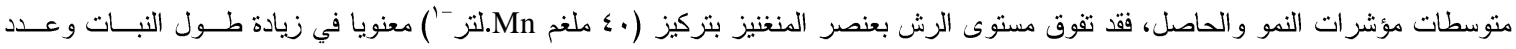

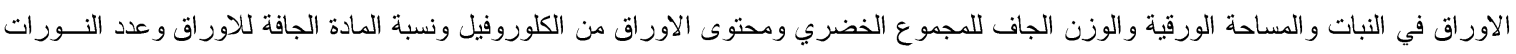

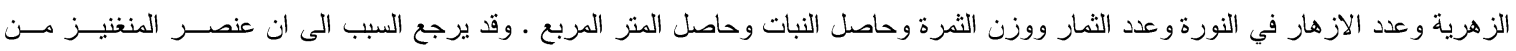

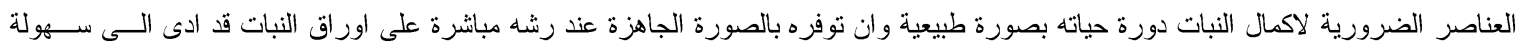
امتصاصه داخل النبات. فهو يساهم في تحويل النترات وتكوين الكلوروفيل والاحماض العضوية و وعليات الاكسدة داخل النبات ومن ثم يساعد في زيادة

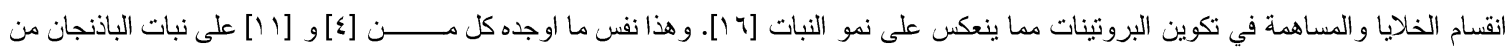

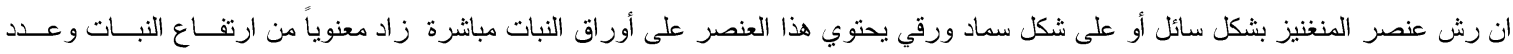

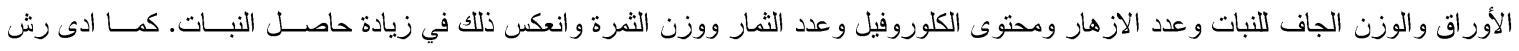

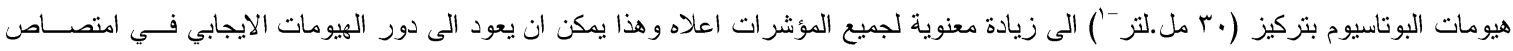

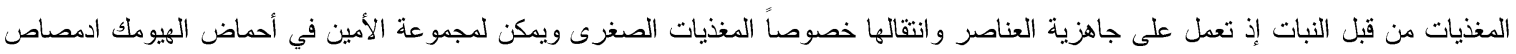

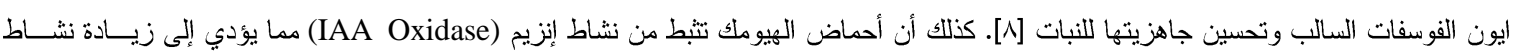

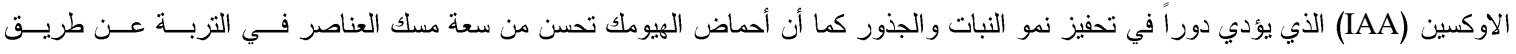

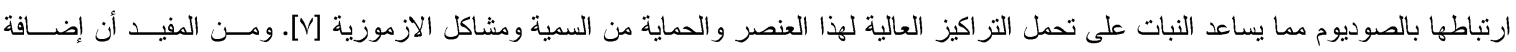

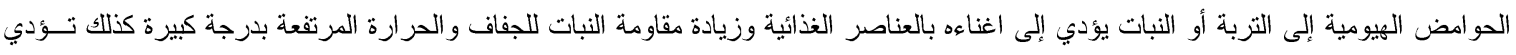

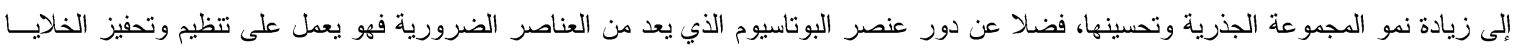

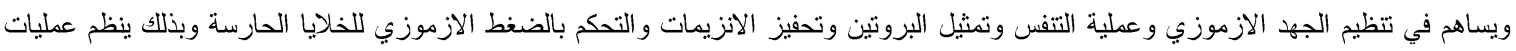

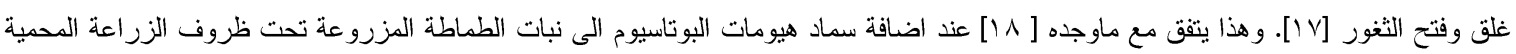
وفي تربة رملية وجود زيادة معنوية في صفات النمو الخضري و الثمري و الحاصل قياسا بمعاملة عدم الاضافة.

\section{CONFLICT OF INTERESTS}

\section{There are no conflicts of interest.}

[1][المحمدي، فاضل مصلح حمادي، الزراعة المحمية. جامعة بغداد، وزارة التعليم العالي و البحث العلمي. بغداد. العراق. بو99.

[2][الصحاف، فاضل حسين رضا. تغذية النيات النطبيقي. وزارة التعليم العالي و البحث العلمي. جامعة بغداد. بيت الحكمة - العراق. 1919

[3][بو ضاحي، يوسف محمد ومؤيد احمد اليونس. دليل تغذية النبات. وزارة التعليم العالي و البحث العلمي . جامعة بغداد. كلية الزراعة.1919 .

[4]التحافي، سامي علي عبد المجيد، حسن علوان سلمان، جابر حمزة عوين. تأثير الرش بالمنغنيز والنحاس في نمو وحاصل الباذنجان صنف بلاكك

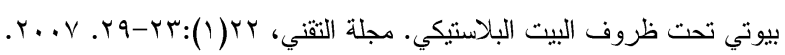

[5]التحافي، سامي علي عبد المجيد، كريم عبد الحسين ردام ، محمد حسين خضير. تأثير عدد الرشات وتركيز السماد الورقي (مايكرونيت هץ) في نمو

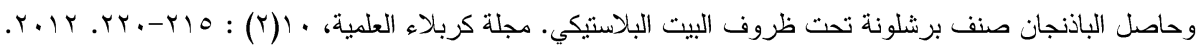

[6] زيدان، رياض وسمير ديوب. تأثير بعض المواد الدبالية والاحماض الامينية في نمو و إنتاج البطاطا العادية . Salanum tuberosum ـ مجلة

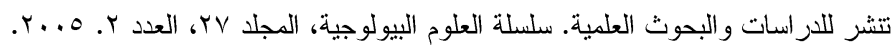

[7]Stevenson, F. J. “Humus chemistry, Genesis, Composition, Reaction,” John wily and Sons, New York. 1994.

[8]Lutzow, M. V.; I. Koegel ; E. Eckschmitt and E. Matzne , “ Stabilization of organic matter in temperate soils mechanism and their relevance under different soil condition-areview, " Eur. Soil. Sci., 57: 426-445,2006.

[9]Ertan, Yildirm," Foliar and soil fertilization of humic acid affect productivity and quality of tomato ", Plant Soil. Sci., 57(2): 182-186,2007. 
[10][الساعدي، ميسون موسى كاظم. تأثير الرش بسماد هيومات البوتاسيوم في نمو وحاصل نبات الطماطة . Lycopersicon esculentum Mill]

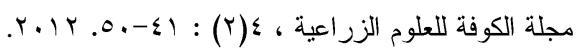

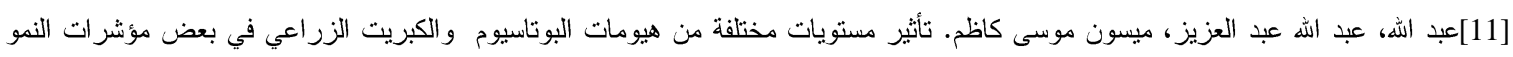

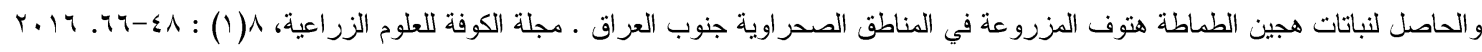

[12]Jackson, M.L , “Chemical Analysis. prentice” Hall Inc. Englewood cliffs. N.J. 1958.

[13]Black, C.A.Ed. Methods of Soil Analysis. Part 2.Amer.Soc. Agro. Madison, Wisconson.USA.1965.

[14]الراوي، خاشع محمود وعبد العزيز محمد خلف اللة. تصميم وتحليل التجارب الزراعية. كلية الزراعة والغابات. جامعة الموصل. العراق.

[15] S.A.S.,. SAS, Users Guide for Personal Computers. Release 7.0. SAS Institute Inc., Cary, NC., USA. (SAS = Statistical Analysis System). 2004

[16]Mengel, K. and E.A. Kirkby, Principles of Plant Nutrition. $4^{\text {th }}$ Edition,International potash institute, IPI, Bern, Switzerland, 685p., 1987.

[17] Marschner, H, Mineral Nutrition of Higher plant .2nd Ed. Academic press, London. England,1995.

[18]الجبوري، رزاق كاظم رحمن. تأثثر السماد العضوي و البوتاسيوم في النمو الخضري والحاصل لنبات الطماطة في الزراعة المحمية غير المدفأة

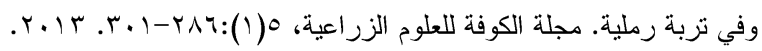

